\title{
Design and Implementation of SPC Systems in Defense Industry Manufacturing Process
}

\author{
Ismar Hrvačić \\ International University of Sarajevo, Faculty of Engineering and Natural Sciences
}

\begin{abstract}
In this paper Statistical Process Control (SPC) was proposed for manufacturing process of a defense product manufacturing company, with the aim of improvement product quality. The production process was monitored from material input to the final output. The processes were mapped and SPC system was proposed. The system was tested by collecting data at the collecting points and analyzed by appropriate SPC tool such as: $\bar{X} \mathrm{R}$ chart, $\mathrm{X} S$ chart and the p-chart. The results show that the processes in the some parts in production are in the under control, processes are capable, but not centered, also some sub-processes of production are not in the control but also not capable.
\end{abstract}

Production process, statistical quality process, specification limits, process capability, process control, control limit, control chart

\section{Corresponding Author:}

\section{Ismar Hrvačić}

International University of Sarajevo,

Hrasnicka cesta 15, 71210 Sarajevo, Bosnia and Herzegovina

Email: ismar2711@gmail.com

\section{Introduction}

Statistical process control is an industry standard methodology for measuring and controlling quality in the production process. SPC methods, good organization and backed management, provide objective means of controlling quality in any organization [1]. Quality data in the form of Process measurements are obtained in real-time during production. In the companies, where the production is on high level and production demand a high level of stability the companies uses the SPC tools. SPC is also a strategy for reducing variability, the cause of most quality. The variation in the process production usually performs a lot of waste or rework [2].

This article is based on statistical quality improvements and making the Control Charts. By the Control Chart the data are monitored the variability of strategic features or Critical to Quality (CTQ) [3]. In the time where the price of manufacturing is in the constant increasing, the company decisions must be based on the facts [4]. These Control Charts in the production have a huge role for keeping the production stable and without a high difference between operations. Control limits are also determined by the capability of the process where the specification limits are determined by the client's needs. In this article, it will be presented the charts from ammunition of a defense company and their data. In quality management, the main division is on "soft tools" and "hard tools" [5]. In this article main focus it will be on "hard tools" which includes control charts for the controlling the process. If the companies want to upgrade their business and their quality production, they must apply techniques, which is mentioned in the next paragraph [6]. Control Charts should be helpful to the companies to improve their production without high defect parts; also, it can help to reorganize the production etc. Control Charts can't be used in the production before the one series of production is not finish, after one 
series is finished the company have the data which will be implemented using proper statistical tool [7]. The SPC have seven tools for measuring the quality in the production. These tools are: histogram, check sheet, Pareto chart, cause and effect diagram, defect concentration diagram, scatter diagram, control charts [8].

Depending on the data, the charts can be classified as attribute or variable control charts [9].

In this article the main focus is on the control charts, which are compound from the $\bar{X} R, \bar{X} S$ and $p$-chart., this charts are usually used in the manufacturing [9]. These charts will be explained in the detail in the next part in the article. The main purpose to do this article is to shown the dispersion data and how the process goes in the company. For the further work and to be sure why these changes in production are happened, the production process must be checked by cause-effect-diagram and design of experiment. Design of experiment (DOE) is a statistical tool; the one of most popular tool for experiment and this tool use the previous data. DOE tool is wide range of use, from engineering field to the medicine [10].

\section{Methods and tools}

In this modern time where production is on high level and where production requires a quality product with small errors, reduces defective parts, reduces production time, the company uses Statistical Process Control (SPC). In this chasing the quality and improving production and service the all big companies have departments for improving quality and getting the data for SPC [7].

Implementation the SPC in the production, the companies has stable manufacturing and stable service, also with using SPC the companies has high level of quality, and in the end they have the high level and stable of trade. When implementing SPC, all activities and responsibilities should be clearly established. Proper Statistical Process Control starts with data collection, and the analysis should be appropriate for the data collection. Tests or inspections for the quality of product or service are not possible with Control Cards if product is not made first. Everything started with the Shewhart circle, which is com composed from 4 main factors which have to be satisfied.

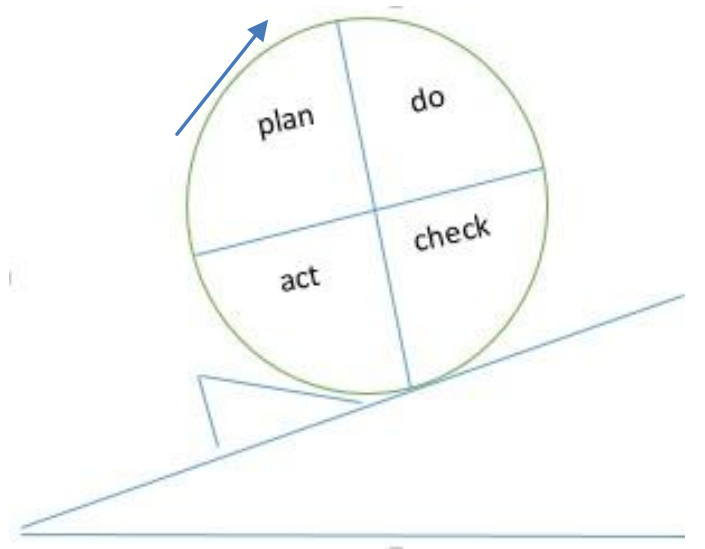

Figure 1: Shewhart circle

InPlan, it is propose what can be change in the system that it can be improve. In Do circle, its role is to accept the propose from the plan part and try it to up to date. In the checking part is part of determining and analyzing the results. Act circle, if change was satisfied, its accepted, but if was not satisfied the results are not implemented and reject. This process usually needed more repetition to solve hard problems. This process should be capable to operate with small difference in variation of product quality characteristics. The seven major tools in SPC are: histogram or stem-and-leaf plot, check sheet, Pareto chart, cause-and-effect diagram, defect concentration diagram, scatter diagram, control chart [8].

In this paper the main focus it will be on Control Charts what was used for determining and solving the problems, and also it was calculated the process capability. The control charts contain the three main border, that is Upper Control Limit (UCL), Lower Control Limit (LCL) and Center Line (CL). Between these lines are distributed the line with points which said about the process, is it in the border or is it in statistical control, nor it is not in the border or it is out of control [6]. 
On the picture below is the schematics shown of Shewhart control chart.

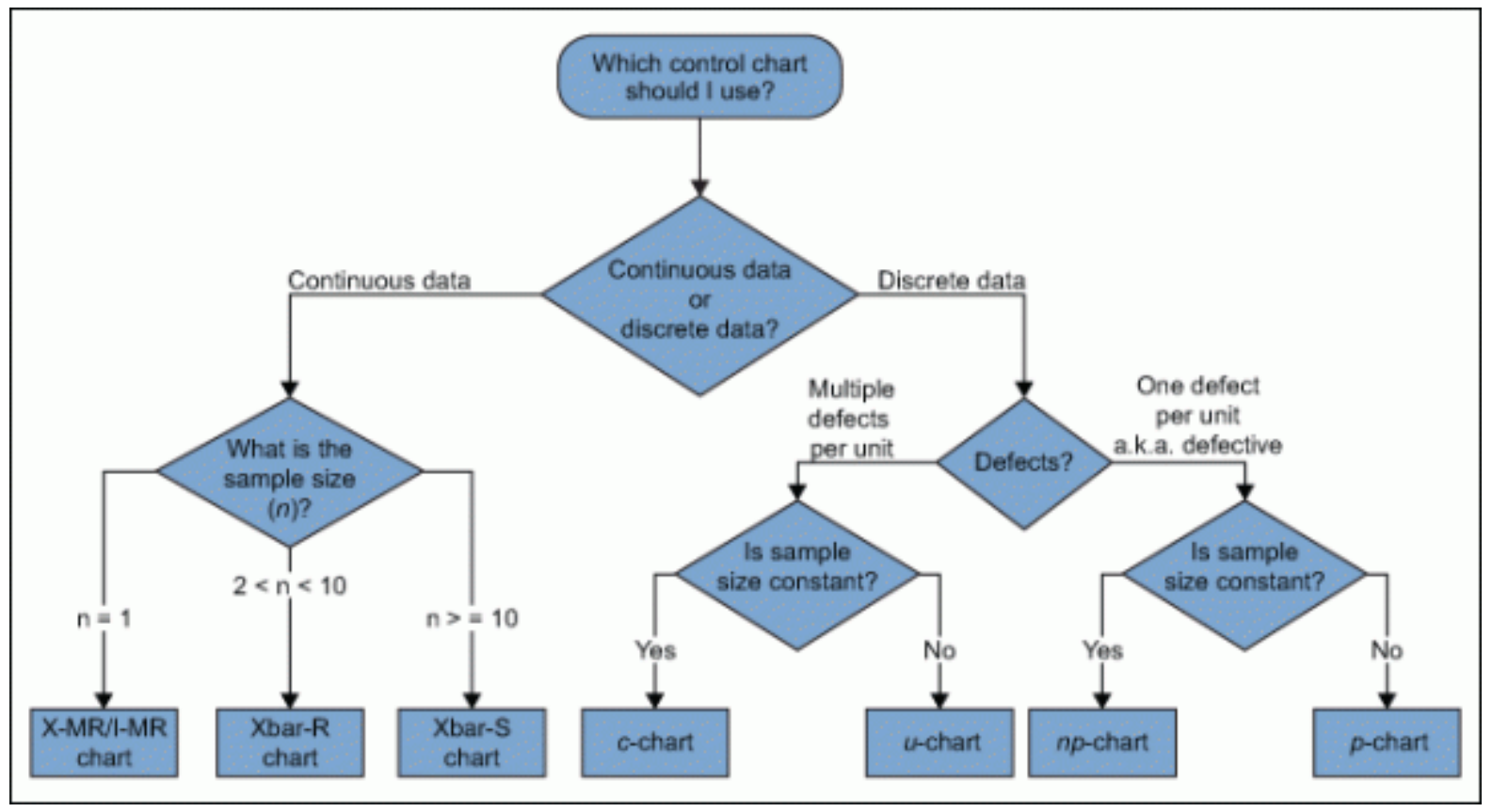

Figure 2: Shewhart control chart[11]

\section{1. $\bar{X} \mathbf{R}$ charts}

The one of control charts which will be used in the further of this report is the $\bar{X} \mathrm{R}$ charts. The $\bar{X} \mathrm{R}$ charts is chart where the calculation is based on the arithmetic mean which represent the X-bar and the range which represent $\mathrm{R}$. The samples are taking from production; the size of sample is between 2 and 10. Below text are the formulas that are needed for calculation $\bar{X} \mathrm{R}$ charts. Also in the legend are explain the meaning of each symbol[8].

$$
\begin{array}{cc}
\bar{X}=\frac{\sum X i}{n} & \begin{array}{c}
L C L x=C L-A^{1} * \bar{R} \\
R=X \max -X \min
\end{array} \\
C L=\frac{\sum \bar{X}}{m} & \operatorname{UCLr}=\bar{R} * D_{4} \\
U C L x=C L+A_{2} * \bar{R} & L C L r=\bar{R} * D_{3}
\end{array}
$$

$\bar{X}$ - average of subgroup; $\bar{R}$ - average range
$A_{2}, A_{3}$ - the factorts for control limits in $\bar{X}$ charts and $\mathrm{R}$ is range
among the samples
$D_{3}, D_{4}$ - the factors in control limits for range charts
$n$ - the sample siz; $m$ - number of sample
$\mathrm{UCL}$ - Upper Control Limit; LCL - Lower Control Limit
$\mathrm{CL}-$ Center Line
$\mathrm{B}_{3}, \mathrm{~B}_{4}$ - the factors in control limits for sample charts

\section{2. $\bar{X}$ and $S$ charts}

In the manufacturing process usually in use is the $\bar{X} \mathrm{R}$ charts but sometimes in use is also the $\bar{X} \mathrm{~S}$ charts. Where $\bar{X}$ also arithmetic mean, and $\mathrm{S}$ is sample standard deviation. This type of the measurements are used when the sample size is bigger than 15 . We have to use the following formulas for calculation $\bar{X} S$ charts [8]:

$$
\begin{array}{cc}
C L=\frac{\sum \bar{X}}{m} & S=\sqrt{\frac{\sum_{i=1}^{n}(X i-\bar{X})^{2}}{n-1}} \\
U C L x=C L+A_{3} * \bar{S} & \begin{array}{c}
U C L s=\bar{S} * B^{4} \\
L C L x=C L-A_{3} * \bar{S}
\end{array} \\
L C L S=\bar{S} * B_{3}
\end{array}
$$




\subsection{P-chart}

A p-chart is an attributes control chart that displays changes in the proportion of defective products, rejects or unacceptable outcomes. It is an indicator of the consistency and predictability of the level of defects in the process. P-charts show how the process changes over time. The p-chart is usually used to monitor the proportion non-comforming (or defectives) in a sample [12]. The process attribute or characteristic is always described in a yes/no, pass/fail, go/no go form. Since a p-chart is used when the subgroup size varies, the chart plots the proportion or fraction of items rejected, rather than the number rejected.

This is indicated by the shifting UCL and LCL lines that depend on the size of the subgroup. For each subgroup, the proportion rejected is calculated as the number of rejects divided by the number of items inspected. When you have a constant subgroup size, use an $n p$ chart instead [12].

\subsection{Zones In Control Charts}

Many of the standard rules examine points based on Zones. The area between each control limit and the centerline is divided into thirds. The third closest to the centerline is referred to as Zone A or $3 \sigma$, the next third is Zone $\mathrm{B}$ is $2 \sigma$, and the third closest to the control limits is Zone $\mathrm{C}$ belongs to $\sigma$. Note that there are two of each of the Zones, one upper and one lower. Zone A is also referred to as the $-3 \sigma$, Zone B is the $-2 \sigma$, and Zone $\mathrm{C}$ is the $-\sigma$. These Sigma zone terms are appropriate only when $-3 \sigma$ is used for the control limits [13].

In the picture below or Figure 3is shown Zones in Control Charts:

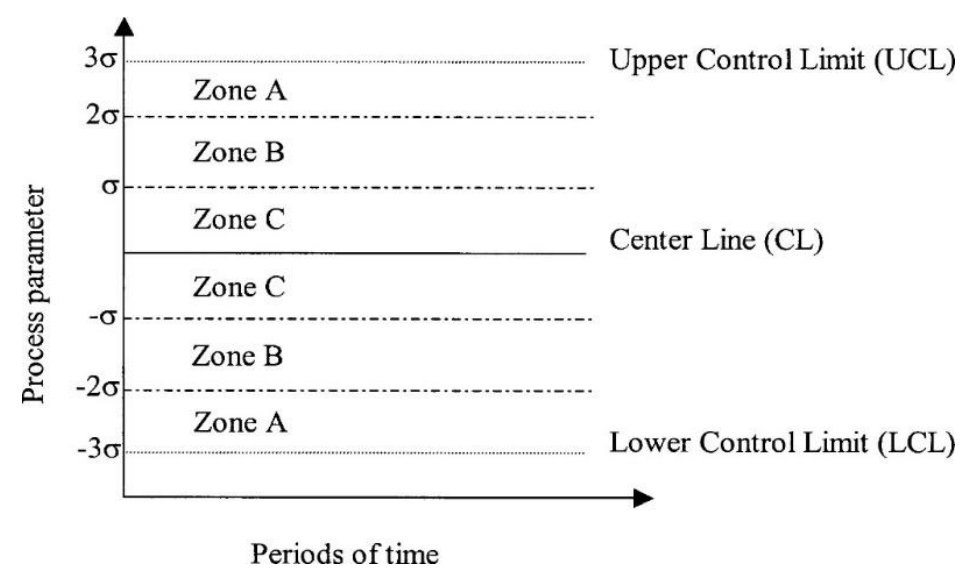

Figure 3: Zones in Control charts

The stable process is the process where all points are in the range of upper specification limit and lower specification limit. The ideal process does not exist because in the process all the time are in the change, as it because of the quality of tools in machine or the material or the machines are not adjustment as good as it needs, but the process must be close as much as possible to ideal. The International Standards Committee is proposed the rules for process controlling stability and that rules are:

- At last 25 points must be in the range of limits,

- Among last 35 points one can go above or below the limits,

- At last 100 points 2 of them can leave the limits.

Also the points can be in the range but process is not stabile and that situations are:

- If 7 points are in the constant increase or constant decrease the process are not stable,

- If 5 points are in constant increase or decrease than process are in critical condition,

- If 7 points constantly are under or above the central line than the process are not stable,

- If 5 points constantly are under or above the central line then the process are critical,

- If 2 out of 3 points are above the range of $2 \sigma$ the process are stable but we need to follow the action,

- If 4 out of 5 points are above or below the $1 \sigma$ this leads to unstable condition. 
Also we need to calculate the process capability and process capability index. The process capability is the important tool in SPC [14]. These information help companies and engineers to see a huge picture about the process [8]. To check whether the process fits or whether the process is out of control it can observed from process capability index $-C_{p}$ with the following formula [15]:

$$
C p=\frac{U S L-L S L}{6 \sigma}
$$

USL -Upper specification limits

LSL - Lower specification limits

$\sigma$ - Standard deviation

The $C_{p k}$ index is about whether the process is centered or not and the formula is:

$$
\begin{gathered}
C_{p u}=\frac{U S L-\mu}{3 \sigma} ; C_{p l}=\frac{\mu-L S L}{3 \sigma} ; \\
C p k=M I N(C p u, C p l)
\end{gathered}
$$

\section{Results and discussion}

In this section of this project, we will discuss the results and explain the all results which wascalculated. Eight statistical Control Charts will be explained. The Control Charts are named in letters because the company strictly banned to use original names, due to sensitive company products.

The rough process configured more than two normal processes and in Figure 4 is shown the Rough Process in the production:

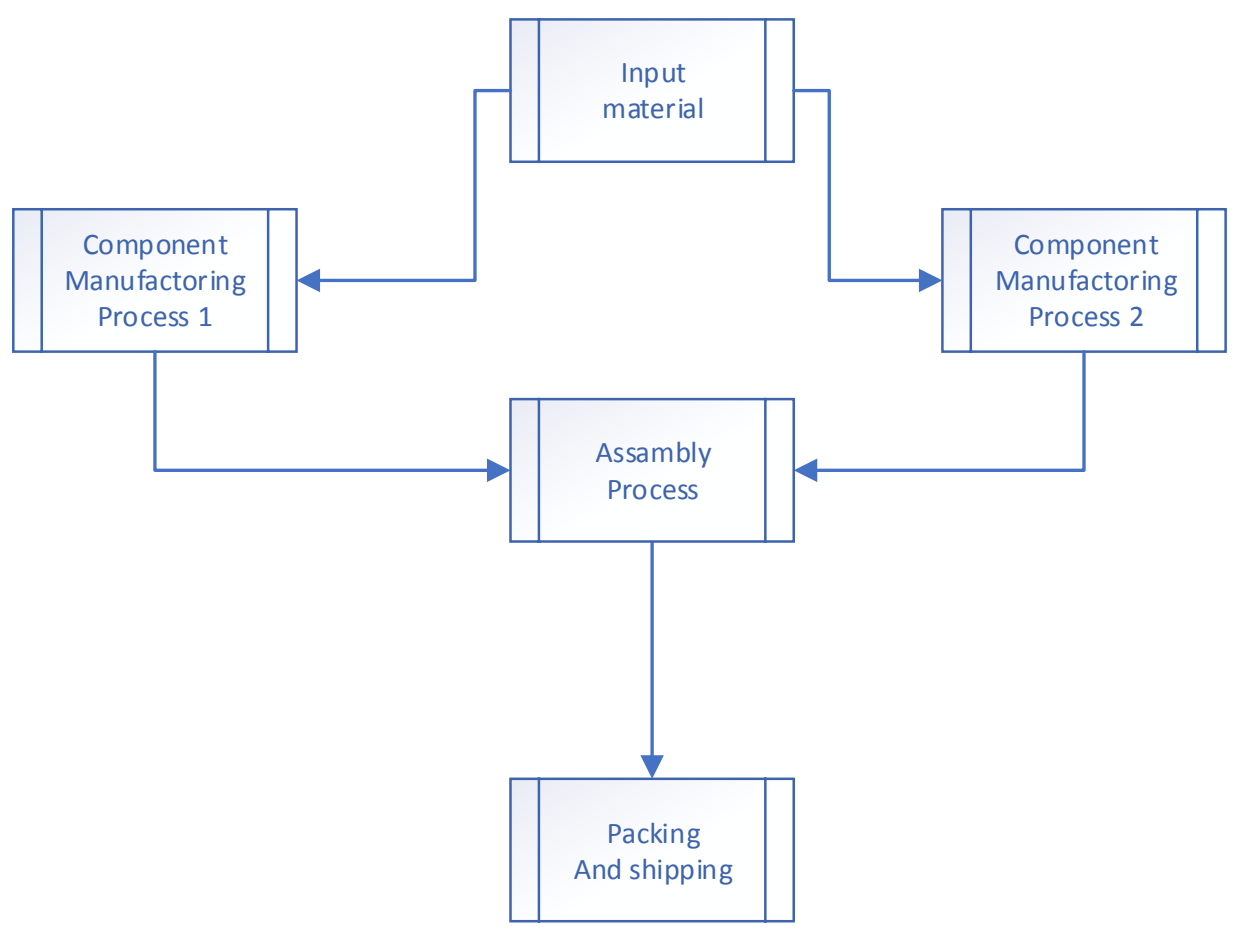

Figure 4: Rough Process Chart[6]

This shown the complete process through the 3 main operations in the production process. In this rough figure from the input the materials go into two manufacturing components and when the processes are done they are go to the final assembly. 
In the Figure 5 it will be shown the process with subprocess of all operations in production.

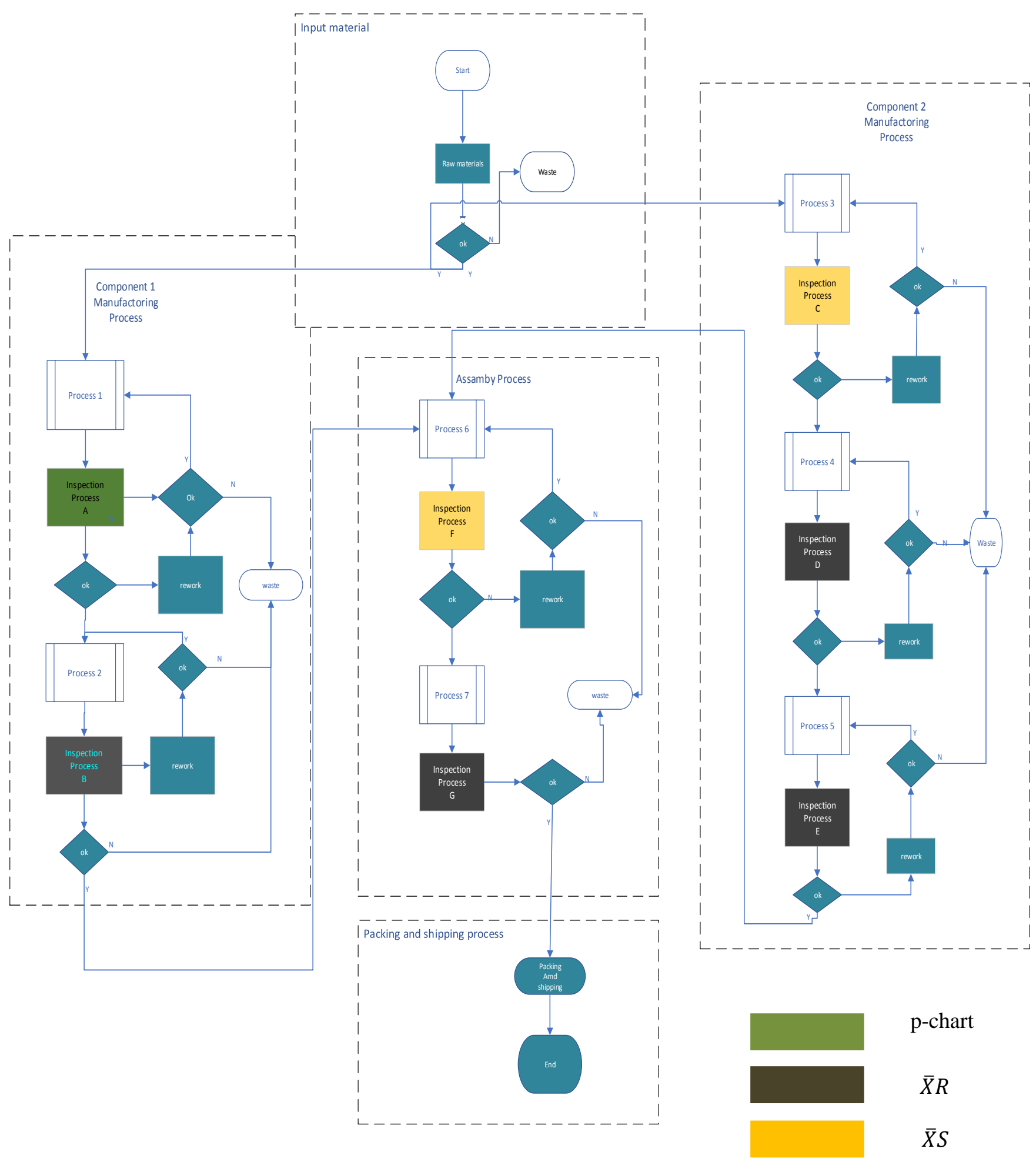

Figure 5: Flow chart for complete production 
Control chart for inspection place A

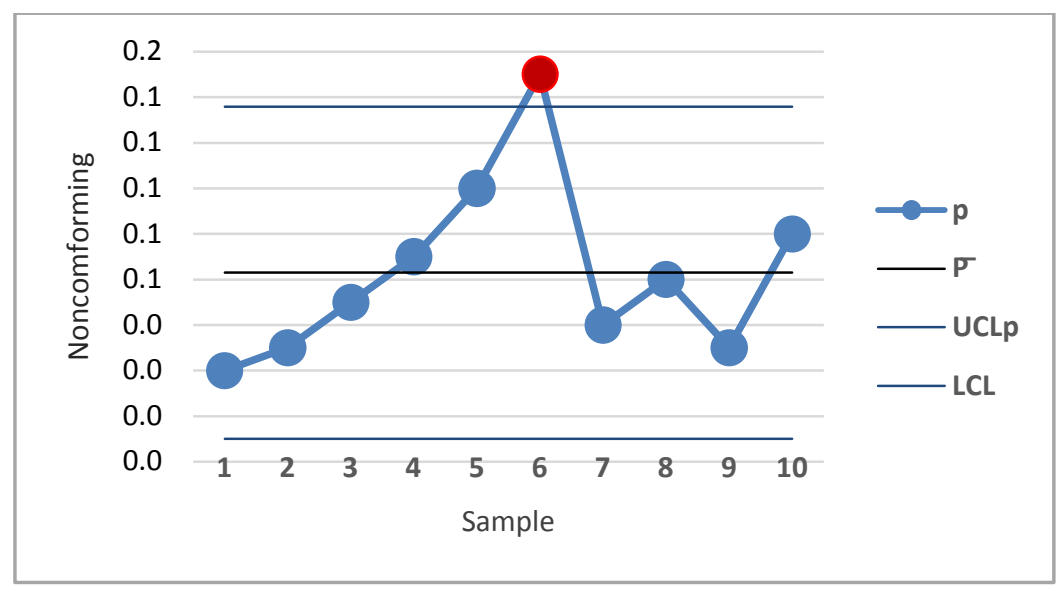

Figure 6: $\mathrm{P}$ - chart for inspection place A

From this chart it can be seen that a process with GO NO GO technique has only one point on above the $\mathrm{UCL}$, and it can be redone. This point is marked with red color and it is onpoint 6 at value 0.2 .

Control chart for inspection place B

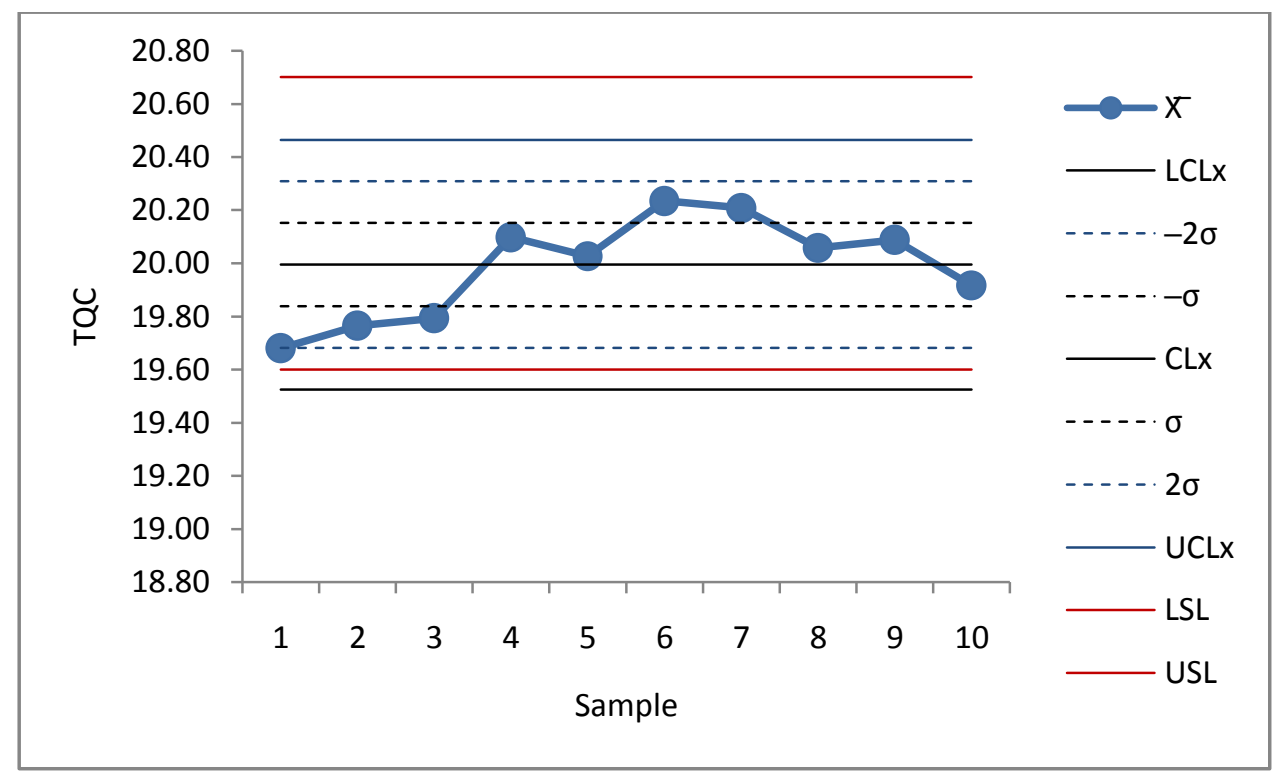

Figure 7: $\overline{\mathrm{X}}$-chart for inspection place $\mathrm{B}$

In this Chart B the process is stable but not confident. The point 1, 2, 3, 6 and 7 are also in the border between UCL and LCL but are in the range. Point 1 at value 19.68, point 2 at value 19.77, and point 3 at value 19.84 are below CL and zone C. The point 6 at value 20.24 and point 7 at value 20.21 are above CL and zone C. In the points 1,2, 3 the order is in increasing, but in the points 6 and 7 is in decreasing. The point 4, 5, 8,9 are in the zone $\mathrm{C}$. This process can lead to unstable control. As the rule said that if is in the Chart 4 of 5 points out of one sigma line the process is not stable and it is critical. Using the formula for $C_{p}$ and $C_{p k}$ it is obtain that $C_{p}$ is 1.2 which indicate to us that process is capable, and the $C_{p k}$ is 0.84 which means these process are not in the center. From the rules the bottom bored for process to be capability stabile is to be $>1.33$, but process are capable also when is $C_{p}=1$ but not stable. 
In the next chart is shown the range of data which are in the border between UCL and LCL also in the zone C. And there is no need for any adjustment.

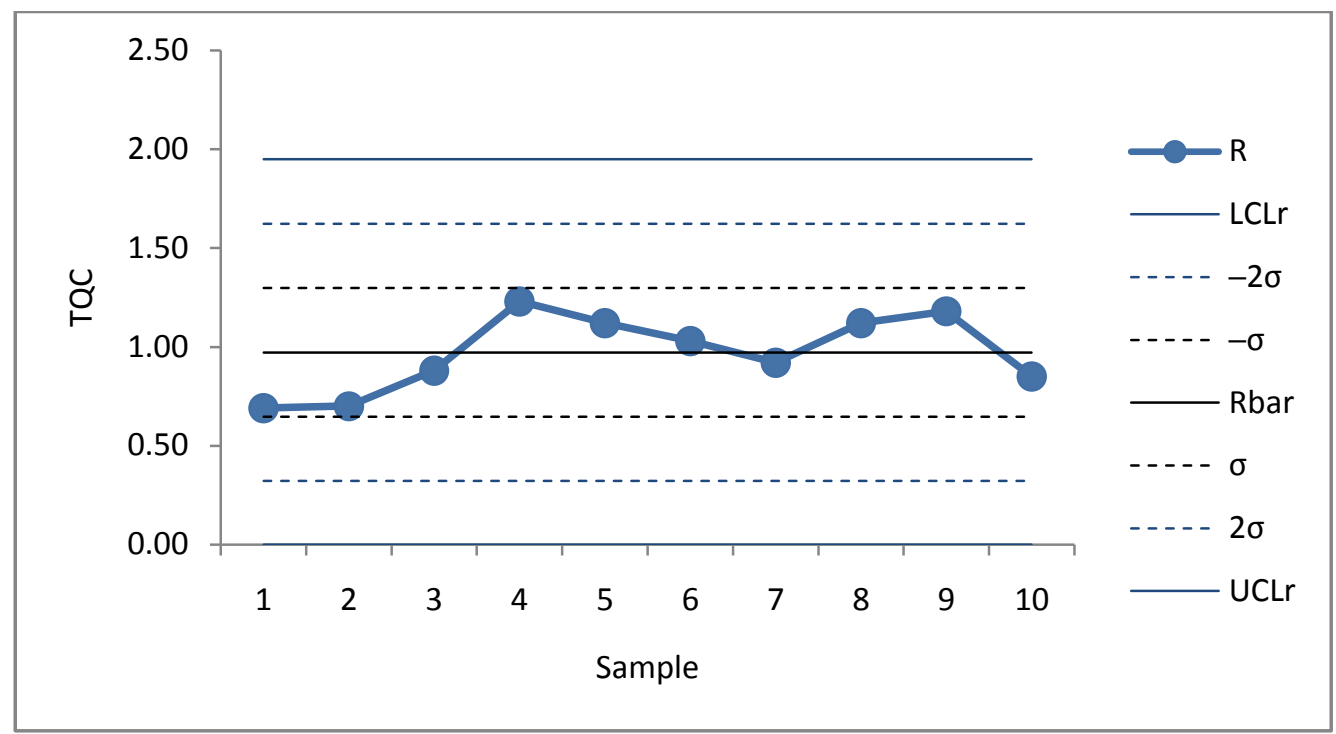

Figure 8: R - chart for inspection place B

The process is not in any of the condition which was mention in the Section 2.4.

Control chart for inspection place C

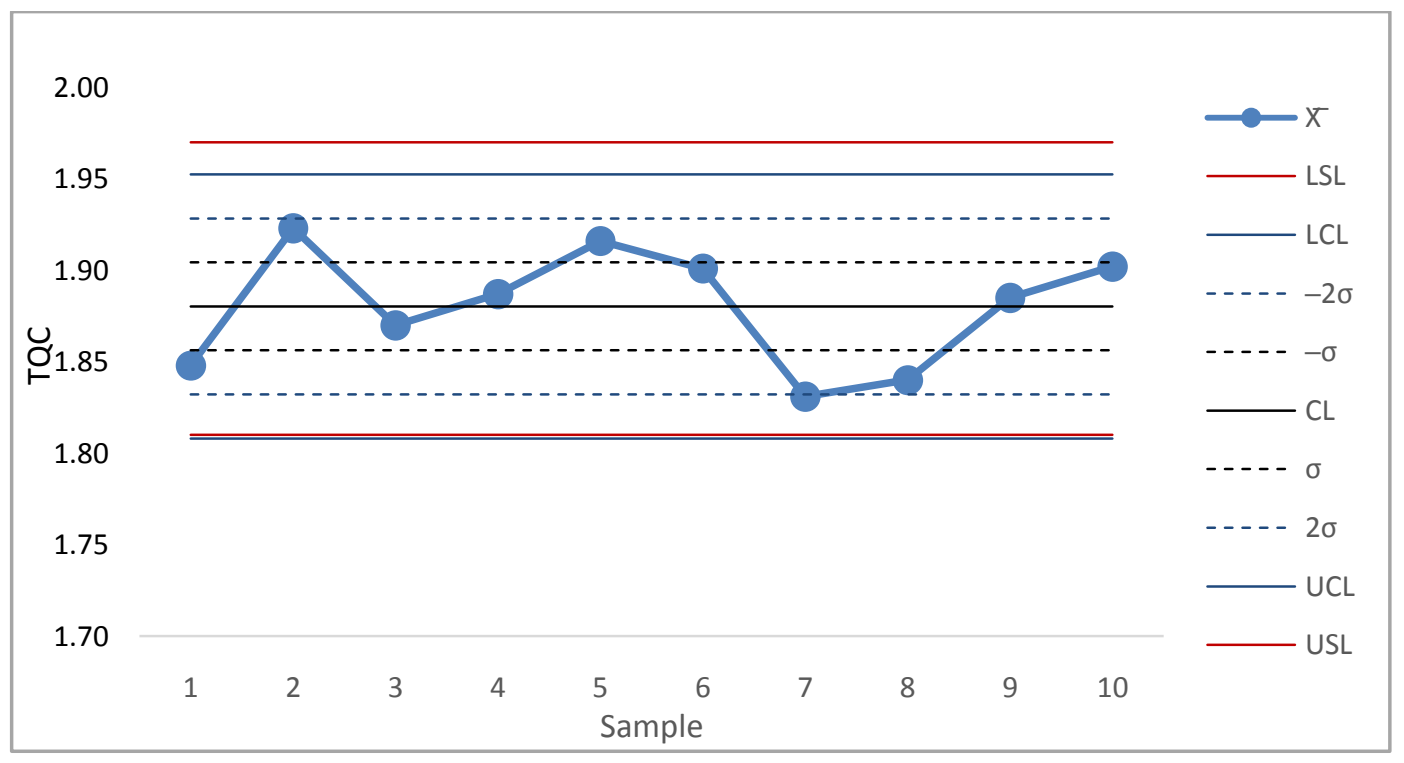

Figure 9: $\bar{X}$ - chart for inspection place $\mathrm{C}$

In the $\bar{X}$ chart the results are in the range of the UCL and LCL, the point 2 at value 1.92 , point 5 at value 1.90 and point 7 at value 1.83 are in the range of limits, card shown the stability of process but it can be unstable. The points 1, 3, 4, 6, 8, 9 and 10 are in the zone C. This process are stable, but the points above the $\mp \sigma$ can be because of the change of material or tool in the machine. In this graph the point 1 and 8 are below and the point 2 is above one sigma zone or zone $\mathrm{C}$, the point 5 is above and the point 7 is below the two sigma zone or zone $\mathrm{B}$, and the process are stable but it can be problematic. 
As it is shown in the graph the process is stable but problematic, refers to the formula for $C_{p}$ and $C_{p k}$ it is obtain that $C_{p}$ is 1.1 which indicate to us that process is capable but not so stable, and the $C_{p k}$ is 1 what indicatesthat process are centered.

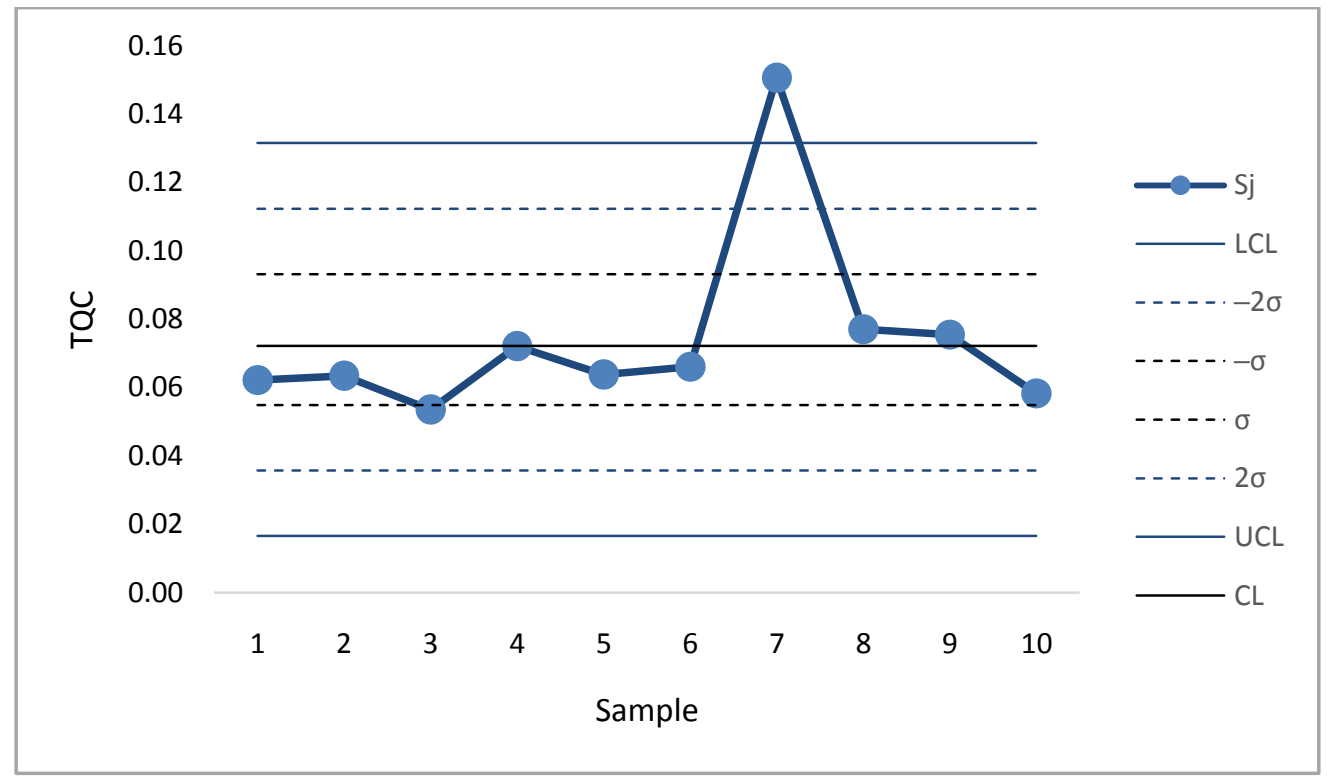

Figure 10: S - chart for inspection place $\mathrm{C}$

In this Charthas only one point above the limits and this card should be adjusted. The difference is on point 7 , at value 0.14 . Also the first 6 points are constantly below the $C L$, the process in the range is not stable, it is critical.

Control chart for inspection place D

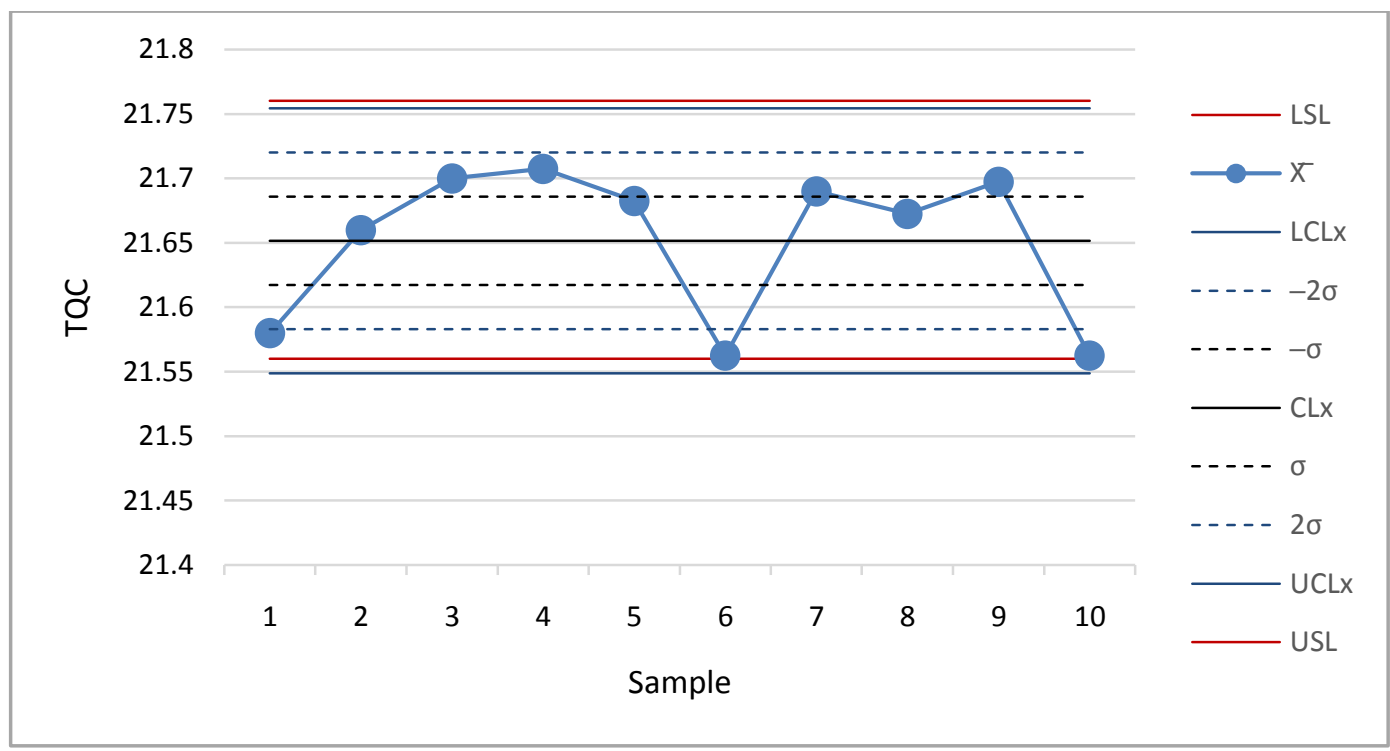

Figure 11: $\overline{\mathrm{X}}$-chart for inspection place $\mathrm{D}$

Control chart for inspection place D is also the $\bar{X} \mathrm{R}$ Chart. In this graph the points $1,6,10$ are below the $2 \sigma$ zone and that is indicate that process are not stable, the company needs to follow these operation. The points $3,4,7,9$ are points below the $1 \sigma$ zone and this leads to unstable condition.

In charts before also are calculated $C_{p}$ and $\mathrm{C}_{\mathrm{pk}}$, in this case the $\mathrm{C}_{\mathrm{p}}$ is 1 and $\mathrm{C}_{\mathrm{pk}}$ is 0.89 , which means that process is capable but not stable, and not centered. 


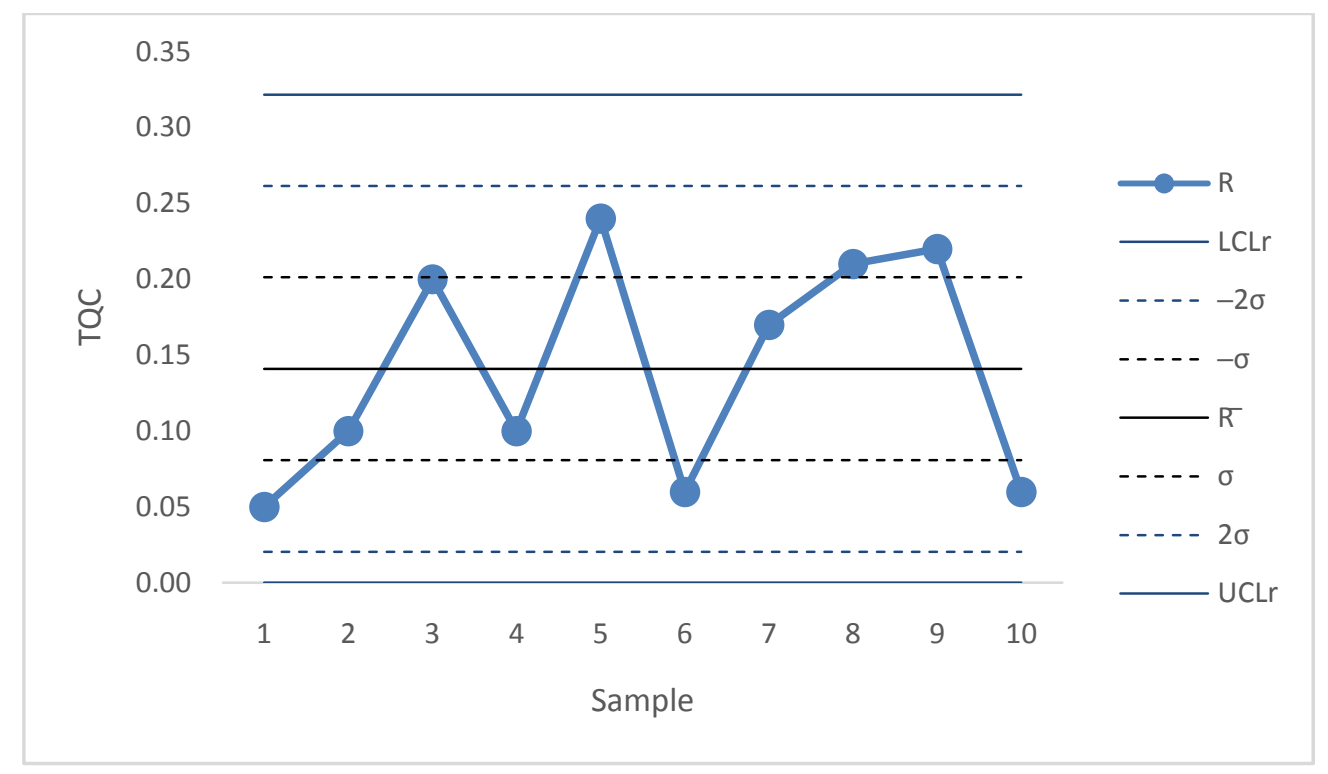

Figure 12: R-chart for inspection place D

This is R-chart and from the rule which was mention in the Chapter 2 Section 2.4 these graph leads to unstable condition, because in the graph 6 points are out of the $1 \sigma$ zone.

Control chart for inspection place $\mathrm{E}$

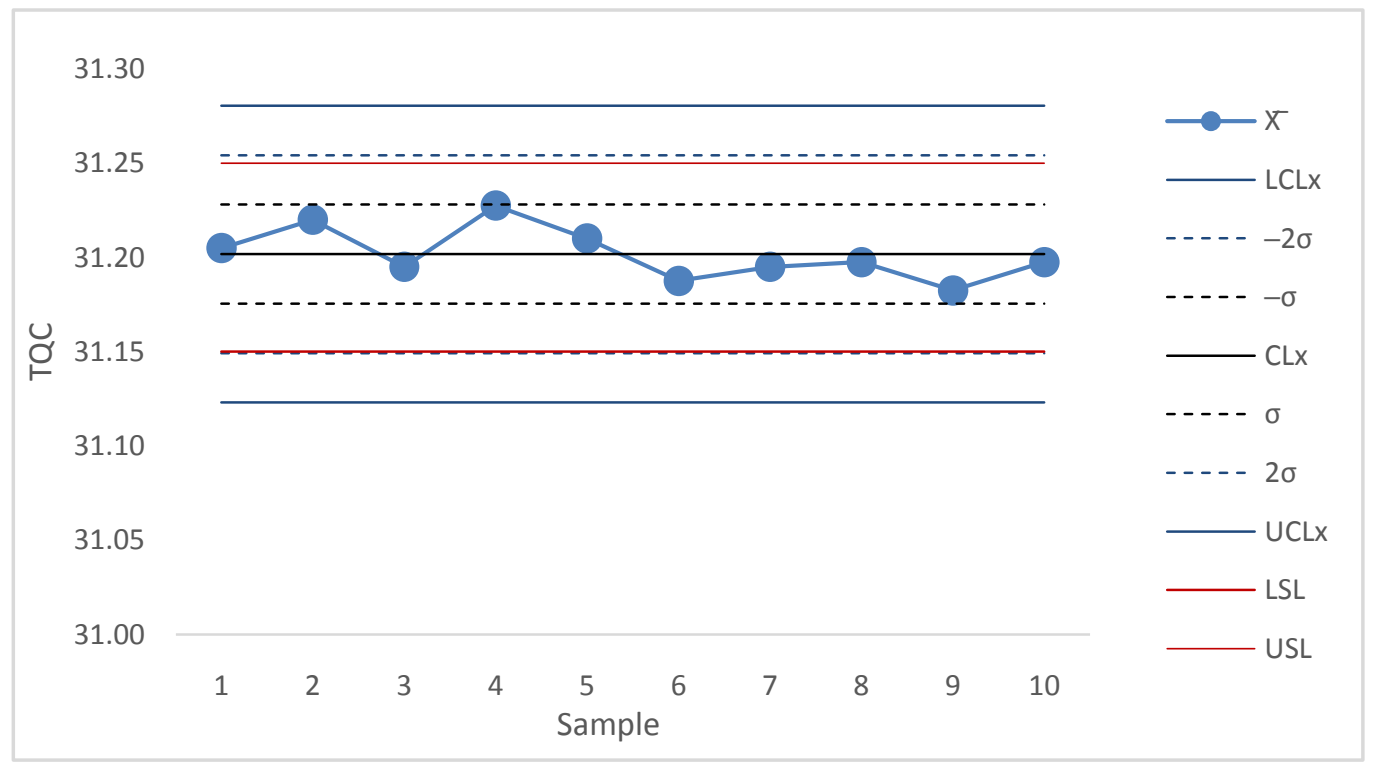

Figure 13: $\overline{\mathrm{X}}$ - chart for inspection place $\mathrm{E}$

In this graph, 5 points are constantly below the center line, this process is still stable but with critical tendency. The calculation for capability process shows that the process is not capable andalso usnot centered, because the results for $C_{p}$ is 0.6 and for $C_{p k}$ is 0.6 . 


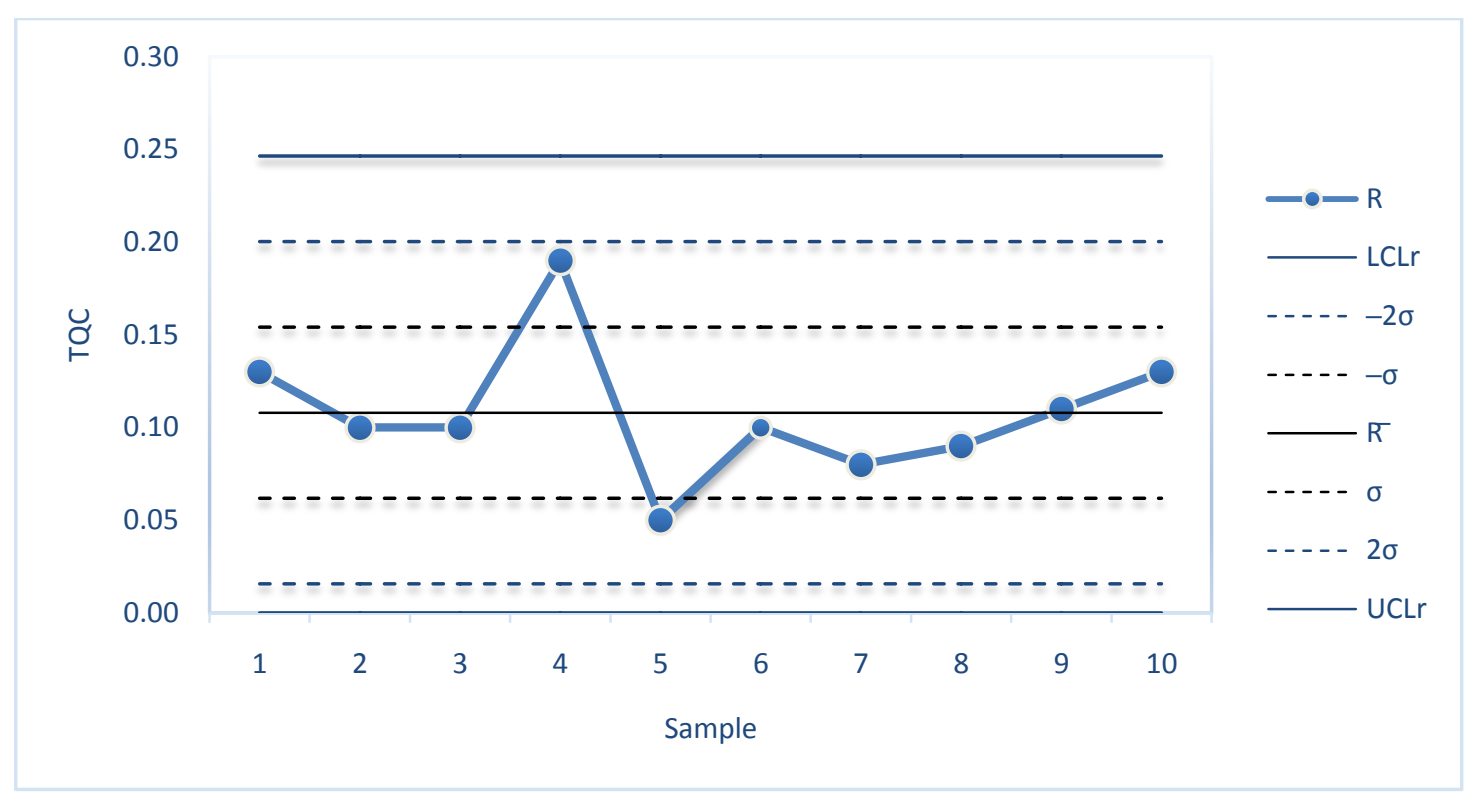

Figure 14: R-chart for inspection place E

In the Range graph we could see that 2 points are out $2 \sigma$ zone and the rule said that if 2 points is out, that process is stable. This is happened because of changing of materials, tools in the machine and adjustment equipment in the machine.

Control chart for inspection place $\mathrm{F}$

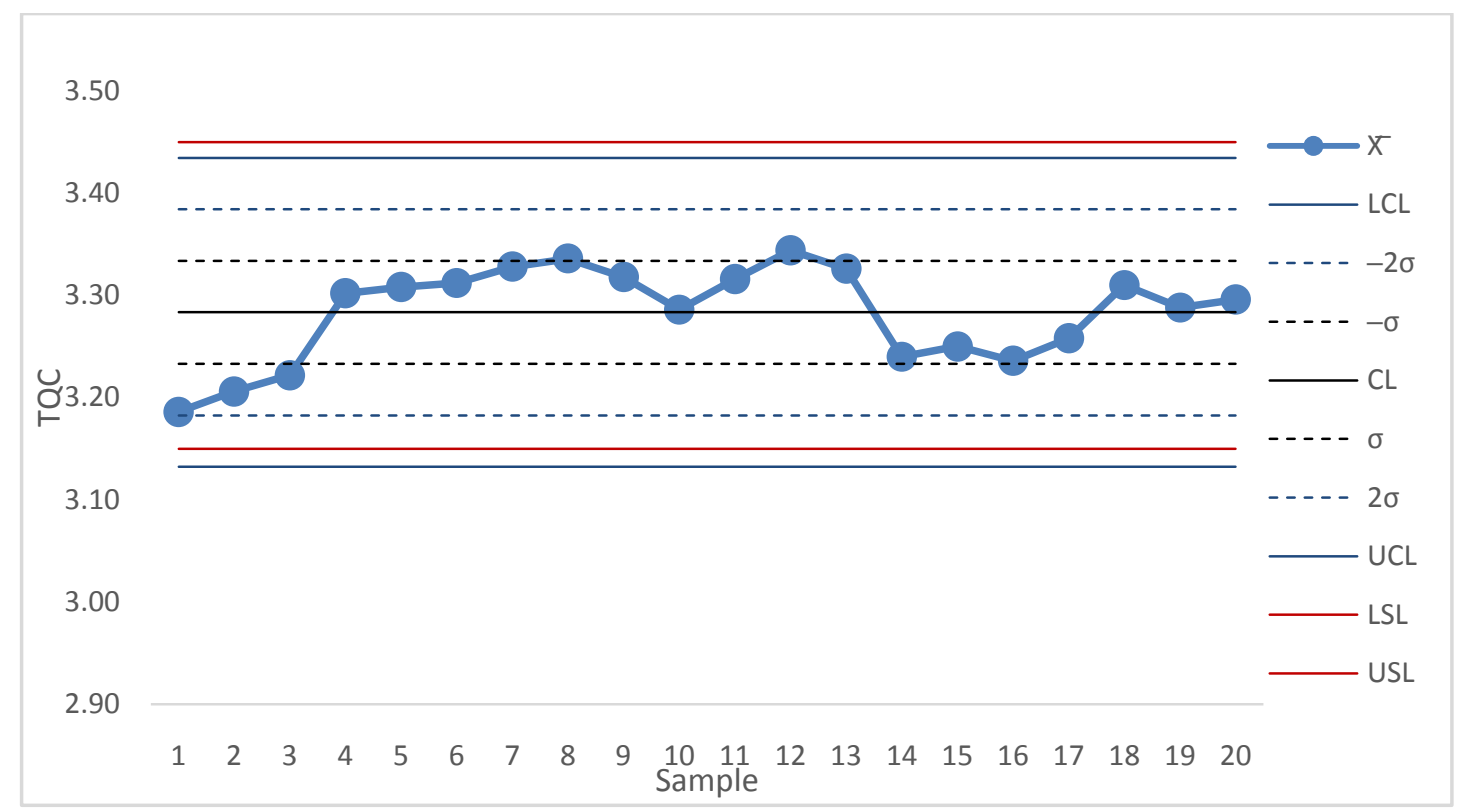

Figure 15: $\overline{\boldsymbol{X}}$-chart for inspection place $\mathrm{F}$

In the $\bar{X}$ chart the 3 points are out of $1 \sigma$ zone on the values at point 1 the values is 3.19 , and the point 2 the values is the 3.22, and the point 3 at value 3.22. This figure shows that process is stable, but process is not centered and must be adjusted. From point 4 to the point 13 all points are above the center line and this is indicator that process are not stabile, this means that process is moved. Also to refer in $\mathrm{C}_{\mathrm{p}}$ and $C_{p k}$ the results 
shown that process is capable but not stable and not centered, so this needed to be adjusted. The $C_{p}$ is $1.0, C_{p k}$ is 0.9 .

In the Figure 16 is shown the $\mathrm{S}$ chart.

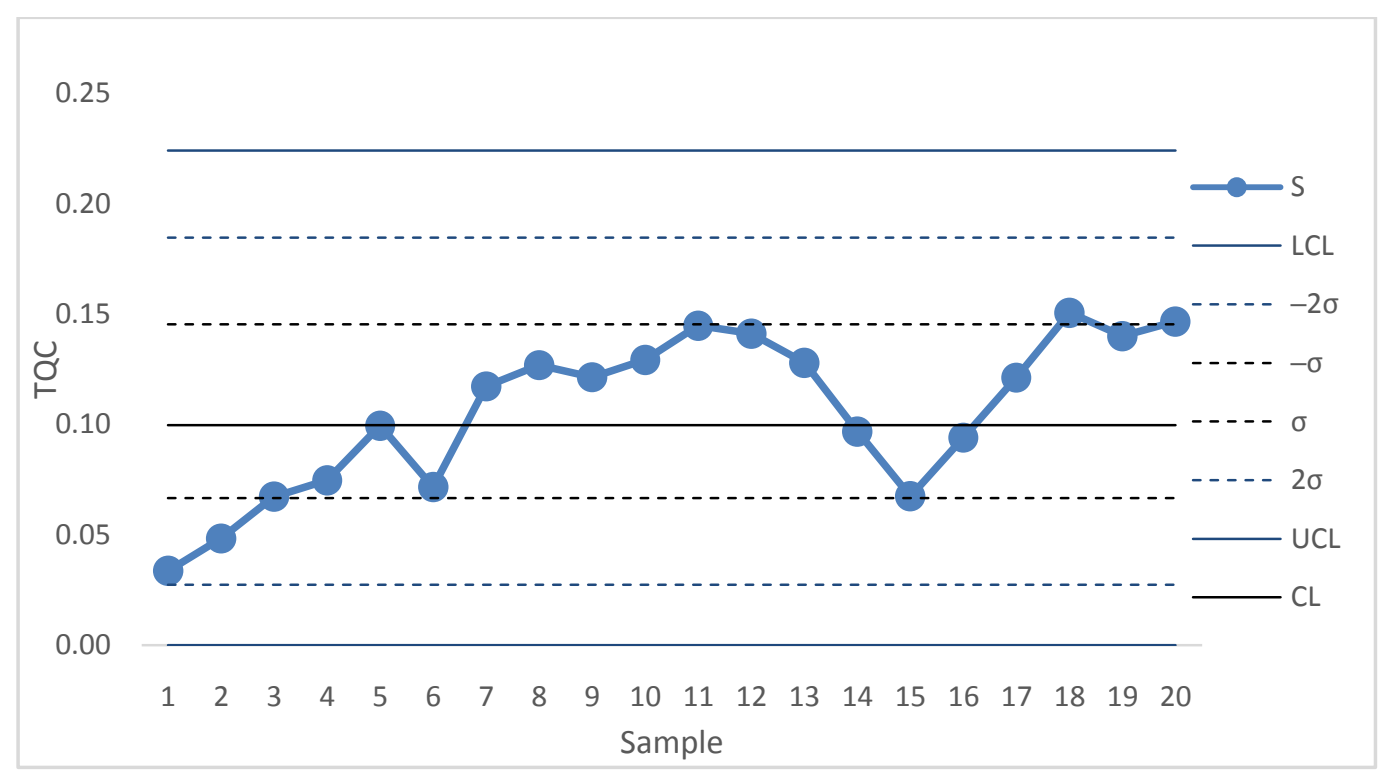

Figure 16: S-chart for inspection place $\mathrm{F}$

In this $S$ chart the results in the beginning 5 points are in constant increase and this leads to unstable process. Also first 6 points are below the center line and this shown that process is critical. From the point 7 points to 13, each point are above the center line and the process is not stable.

\section{Control Card data G}

In this Chart we can see that process is in the control.

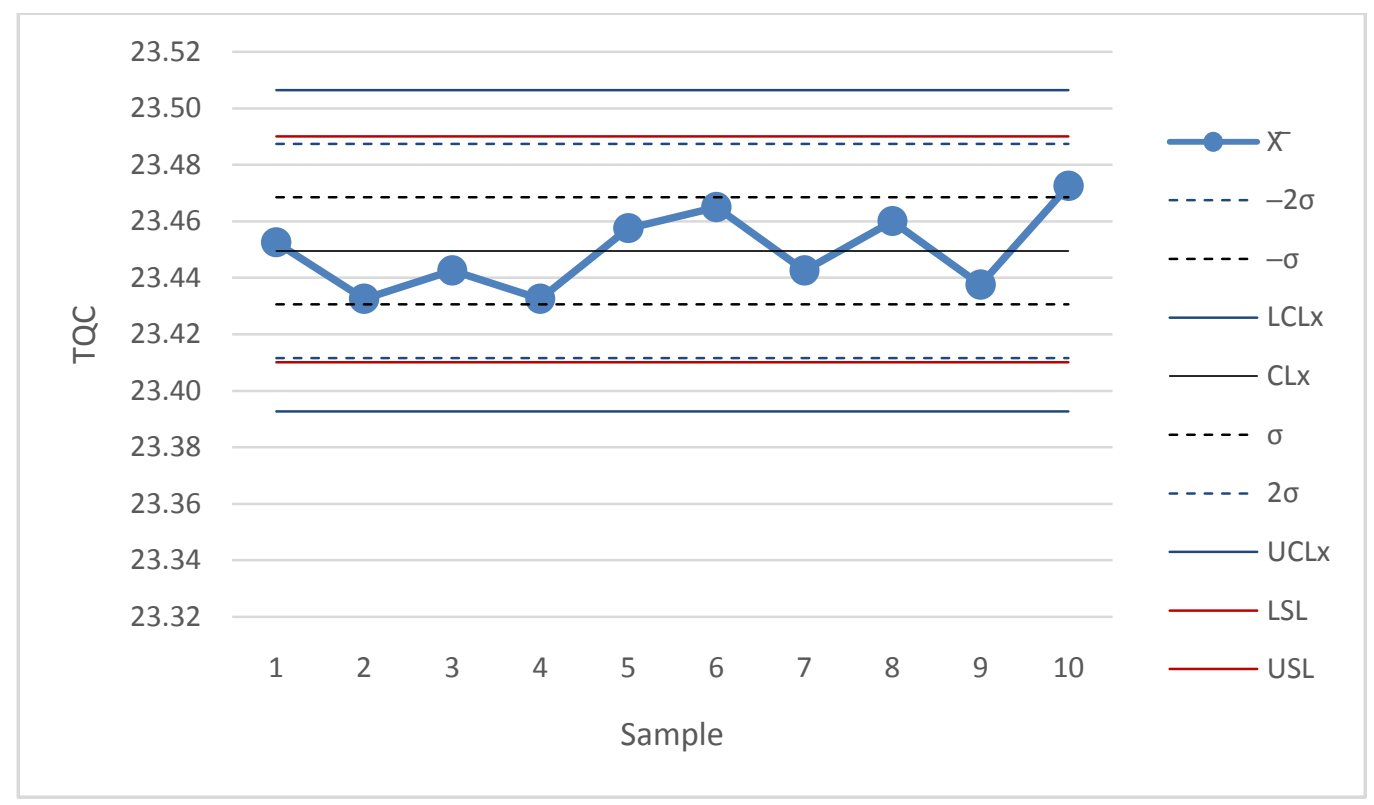

Figure 17: Chart $G-\bar{X}$ chart for inspection place $G$ 
From this chart we can see the results in the $\bar{X}$ chart the data distributed among the central line. In this case, it cannot apply the rule form Chapter 2.From calculation for $C_{p}$ and $C_{p k}$ the conclusion is that process is not capable and not centered. The results for $C_{p}$ is 0.7 and for $C_{p k}$ is 0.69 .

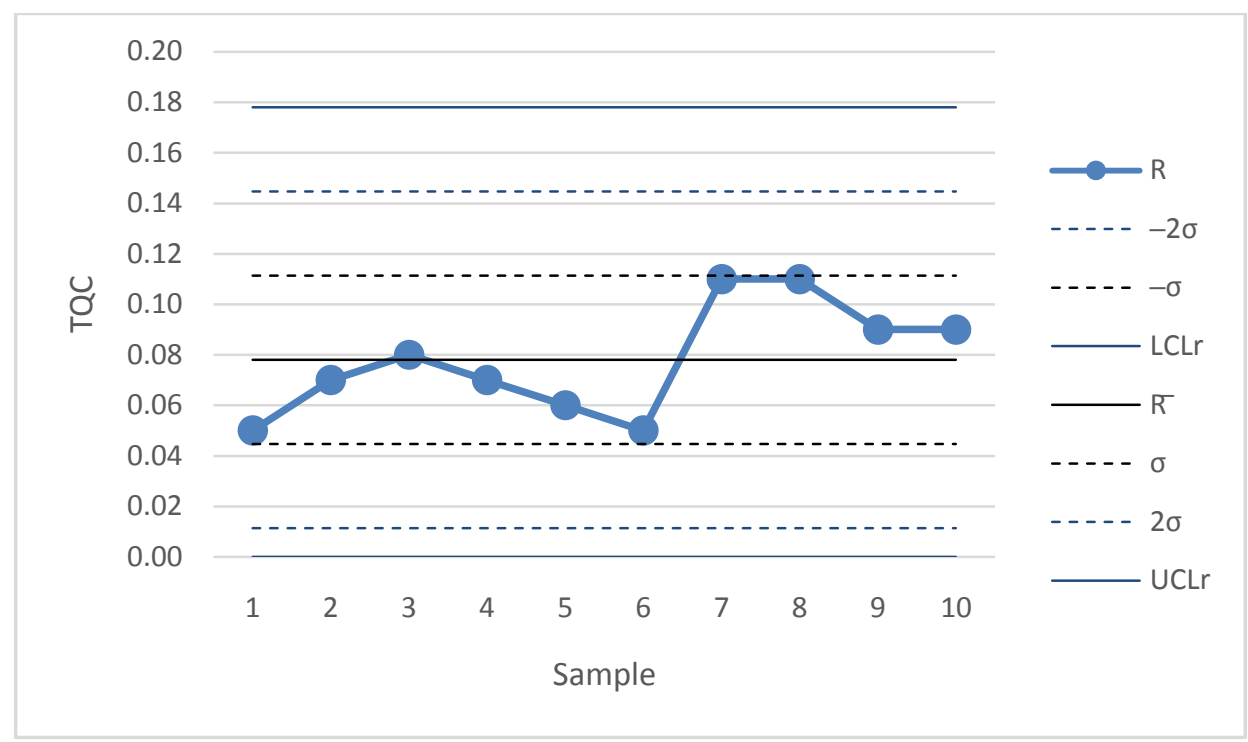

Figure 18: R-chart for inspection place $\mathrm{G}$

In this $R$ chart cannot be applied the rules from chapter 2 . This process is in the control.

To identify potential caused of nonconforming parts DOE can be used as a tool. In addition, using Pareto analysis will give an input to focus on most frequent types of nonconformities. Identifying an en eliminating the main cause of non conforming parts may reuce the up to $80 \%$ of waste [16].

\section{Conclusion}

The Control Charts are become the main tool for following the quality of production in the huge production companies. The statistical data are also indicator for quality of some product. In engineering sense the statistical charts are become the main tool for to conduct and keep process in statistical control. With this charts the production can be easily adjusted and process become more stable. It was shown that process is mostly capable but not stabile. On the other hand, there are a few cases where process is not capable and not centered. When the control charts are in the optimal usage, it assures the process are in constantly monitoring and secure, and that product will be easier to repair if goes something wrong. All the research what was provide and research all aspect of controlling the production in the company and data what was received from them are in the range of satisfying.In the processes which are out of control and processes which are not capable and centered must be examine by DOE or cause effect-diagram, which use previous data to figure why these anomalies occurred.From the previous experience, these anomalies usually occur because of tool changing in the machine, bat materials, etc. The small correction it should be in control chart where the data are out of control limits, if these operations can be reworked.

\section{References}

[1] J. S. Ockland, Statistical Process Control, 6th Edition, 2008.

[2] E. H. S. Oberoi, M. Parmar, H. Kaur and R. Mehra, "SPC (Statistical Process Control): A Quality Control Technique for Confirmation to Ability of process," International Research Journal of Engineering and Technology (IRJET), vol. 03, no. 06, pp. 666-672, 2016.

[3] A. G. Maugeri and G. Arcidiacono, "Efficient Determination of Sampling Rate and Sample Size in Statistical Process Control," Universal Journal of Industrial and Business Management, pp. 103-110, 
2014.

[4] I. Madanhire and C. Mbohwa, "Application of Statistical Process Control (SPC) in Manufactoring Industry in Developing Country," in 13th Global Conference on Sustainable Manufacturing - Decoupling Growth from Resource Use, 2016.

[5] B. Durakovic, H. Bašić and H. Muhić, "The Interrelationships between Quality Managment Practicies and their Effects on Innovation," Trends in the Development of Machinery and Associated Technology, Budapest, pp. 181-184, 2014.

[6] V. D. M. T. V. ŠIBALIJA, "SPC and Process Capability Analysis- Case Study," Total Quality Management \& Excellence, vol. 37, pp. 1-6, 2009.

[7] B. Duraković and H. Bašić, "Textile Cutting Process Optimization Model Based on Six Sigma Methodology in a Medium-Size Company," Jurnal of Trends in the Development Machinery and Associated Technology, vol. 16, pp. 107-110, 2012.

[8] D. Montgomery, Introduction to Statistical Quality Control, $6^{\text {th }}$ Edition, 2003, pp. 177-235.

[9] R. Godina, J. C. Matias and S. G. Azevedo, "Quality Improvement With Statistical Process Control in the Automotive Industry," International Journal of Industrial Engineering and Management (IJIEM), vol. 7, pp. 1-8, 2016.

[10] B. Duraković, "Design of Experiments Application, Concepts, Examples: State of the Art," Periodicals of Engineering and Natural Sciencie, pp. 421-438, 2017.

[11] "http://www.cqeacademy.com/cqe-body-of-knowledge/continuous-improvement/quality-controltools/control-charts/," 20 February 2018. [Online]. [Accessed 2018].

[12] M. Xie, T. N. Goh and V. Kuralmani, Statistical Models and Control Charts for High Quality process, Springer Science + Business Media New York, pp. 4-5, 2002.

[13] [Online]. Available: http://www.statit.com/services/SPCOverview_mfg.pdf. [Accessed 25 January 2018].

[14] Đ. Vukelić, J. Hodolič, T. Vrečič and P. Kogej, "Development of a System for Statistical Quality Control of the Production Process," Facta Universitatis, Series: Mechanical Engineering, vol. 6, pp. 75-90, 2008

[15] B. Duraković and H. Bašić, "Continuous Quality Improvement in Textile Processing by Statistical Process Control Tools: A Case Study of Medium-Sized Company," Periodicals of Engineering and Natural Sciences, vol. 1, pp. 39-46, 2013.

[16] H. Bašić, B. Duraković and A. Softić, "Six Sigma Model Testing In Optimizing Medium-Sized Company Production Process," Journal of Trends in the Development of Machinery and Associated Technology, vol. 16, no. 1, pp. 103-106, 2012. 